\title{
Erratum to: Identification of a Novel Binding Protein of FAT10: Eukaryotic Translation Elongation Factor 1A1
}

\author{
Xin $\mathrm{Yu}^{1} \cdot$ Xiuxia Liu ${ }^{1,2} \cdot$ Tiande Liu $^{1} \cdot$ Kui Hong ${ }^{2} \cdot$ Jun Lei $^{1} \cdot$ Rongfa Yuan $^{1} \cdot$ \\ Jianghua Shao ${ }^{1,2}$
}

Published online: 8 December 2016

(c) Springer Science+Business Media New York 2016

\section{Erratum to: Dig Dis Sci (2012) 57:2347-2354 DOI 10.1007/s10620-012-2189-1}

Figure 5 and the text reported erroneous data regarding the influence of FAT10 on mRNA expression of eEF1A1. The corrected Fig. 5 and caption are depicted here.

Text corrections:

1. Last sentence of Abstract:

Substitute with: "Knockdown of FAT10 with siRNA downregulated eEF1A1 expression at the protein level but not at the mRNA level in human hepatocellular carcinoma cells."

2. Fourth sentence in the final paragraph of Introduction

Substitute with: "Although siRNA knockdown of FAT10 significantly downregulated eEF1A1 at the protein level, eEF1A1 mRNA expression was not affected in HCC cells."

The online version of the original article can be found under doi:10.1007/s10620-012-2189-1.

Jianghua Shao

shao5022@163.com

1 The Department of Hepatobiliary Surgery, The Second Affiliated Hospital of Nanchang University, Nanchang 330006, China

2 The Key Laboratory of Molecular Medicine, The Second Affiliated Hospital of Nanchang University, Nanchang 330006, China
3. Results, third subheading:

Substitute for existing: "FAT10 siRNA Downregulates eEF1A1 Protein Expression, but Does Not Affect eEF1A1 mRNA Abundance."

4. First sentence of the first paragraph after the third subheading in "Results"

Substitute: "affect" for "effect."

5. Final sentence of the first paragraph after the third subheading in "Results"

Substitute with: "In Fig. 5, 0.2-0.3 of the FAT10 expression level was relative to the expression level of FAT10 (ratio to GAPDH). To better display the mRNA expression relationship between FAT10 and eEF1A1, the expressions of FAT10 and eEF1A1 in the control group were, respectively, set to 1 in the redone experiment. Then, the relative expression differences of FAT10 between the shFAT10 group and the control group were calculated by the way of $-\Delta \Delta C_{t}$, while the relative expression differences of eEF1A1 between the shFAT10 group and the control group were also calculated. The results show that, compared with the control group, FAT10 expression in the shFAT10 group is decreased about 5 times, while the eEF1A1 expression remains unchanged. Thus, only FAT10 mRNA was significantly downregulated in the FAT10 siRNA-transfected cells $(p<0.01)$, indicating that FAT10 siRNA did not downregulate eEF1A1 mRNA expression."

6 . First sentence of the second paragraph after the third subheading in "Results"

Substitute: "affect eEF1A1 protein expression" for "effect eEF1A1 expression."

7. First paragraph of the "Discussion"

Substitute last sentence with: "Furthermore, siRNA knockdown of FAT10 significantly reduced eEF1A1 protein expression without affecting the abundance of eEF1A1 mRNA." 
Fig. 5 FAT10 siRNA did not affect eEF1A1 mRNA levels. The siRNA fragment for FAT10 was transfected into Hep3B (a) and MHCC97H (b) cells, and total RNA was extracted for quantitative PCR. $* * p<0.01$
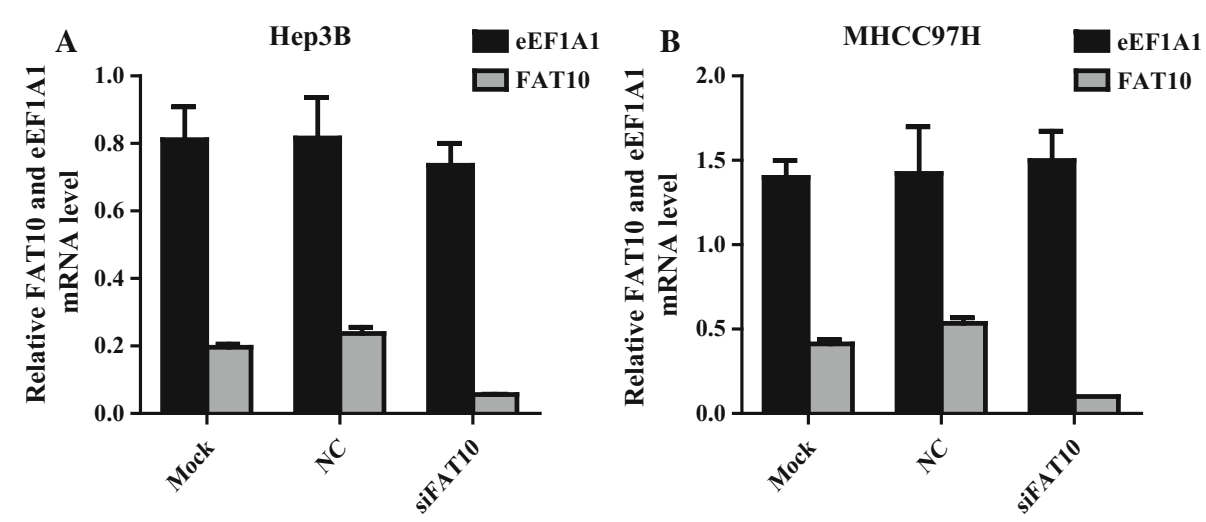

8. Final sentence, sixth paragraph of the "Discussion" Substitute with: "In HCC cells, siRNA knockdown of FAT10 significantly reduced eEF1A1 protein expression without affecting the abundance of eEF1A1 mRNA, confirming that FAT10 is a likely regulator of eEF1A1." 